Published in final edited form as:

Dig Dis Sci. 2019 June ; 64(6): 1651-1659. doi:10.1007/s10620-019-05570-1.

\title{
Vedolizumab Concentrations Are Associated with Long-Term Endoscopic Remission in Patients with Inflammatory Bowel Diseases
}

\author{
Andres J. Yarur ${ }^{1}$, Alexandra Bruss ${ }^{2}$, Snehal Naik ${ }^{3}$, Poonam Beniwal-Patel ${ }^{1}$, Caroline Fox ${ }^{1}$, \\ Anjali Jain ${ }^{3}$, Brandon Berens ${ }^{2}$, Amir Patel ${ }^{1}$, Ryan Ungaro ${ }^{4}$, Bayda Bahur ${ }^{3}$, Marla Dubinsky ${ }^{4}$, \\ Daniel J. Stein ${ }^{1}$ \\ ${ }^{1}$ Division of Gastroenterology and Hepatology, Medical College of Wisconsin, Hub for \\ Collaborative Medicine, 8701 Watertown Plank Road, Milwaukee, WI 53226, USA \\ ${ }^{2}$ Clinical Translational Science Institute, Medical College of Wisconsin, Milwaukee, WI, USA \\ ${ }^{3}$ Prometheus Laboratories Inc, San Diego, CA, USA \\ ${ }^{4}$ Icahn School of Medicine, Mount Sinai Hospital, New York, NY, USA
}

\begin{abstract}
Background-The aim of this study was to assess the relationship of serum vedolizumab concentrations (SVC) during induction and endoscopic remission in patients with inflammatory bowel diseases (IBD) after 52 weeks of therapy with vedolizumab. We also sought to assess the incidence of antibody to vedolizumab (ATV) formation, the effect of ATV on drug pharmacokinetics and efficacy, and identify variables associated with SVC through the first 30 weeks of treatment.
\end{abstract}

Methods-This is a prospective cohort study of patients with active IBD initiating standard therapy with vedolizumab. Collected variables included demographics, clinical disease activity, biomarkers, pre-infusion SVC, and ATV measured at weeks 2, 6, 14, 22, and 30. Primary outcome was steroid-free endoscopic remission at week 52.

Results-Fifty-five patients were included. Patients that achieved steroid-free endoscopic remission by week 52 had higher SVC at weeks $2,6,14,22$, and 30 , but only achieved statistical significance at weeks 2 and 6 . Only 3 out of the 55 study subjects (5.5\%) had detectable ATV through the follow-up. Overall, there were a positive correlation between SVC and serum albumin and a negative correlation with C-reactive protein, fecal calprotectin, and body mass. Vedolizumab concentrations $\geq 23.2 \mathrm{mcg} / \mathrm{ml}$ at week 2 were associated with endoscopic remission at week 52 (OR 8.8 [95\% CI 2.6-29.7], $p<0.001)$.

Andres J. Yarur, ayarur@mcw.edu.

Electronic supplementary material The online version of this article (https://doi.org/10.1007/s10620-019-05570-1) contains supplementary material, which is available to authorized users.

Publisher's Note Springer Nature remains neutral with regard to jurisdictional claims in published maps and institutional affiliations. 
Conclusions-Vedolizumab concentrations during induction were associated with endoscopic remission at week 52. Interventional studies looking into improved efficacy with higher drug exposure are warranted.

\section{Keywords}

Vedolizumab; Vedolizumab levels; Anti-vedolizumab antibodies; Mucosal healing; Therapeutic drug monitoring; Crohn's disease; Ulcerative colitis

\section{Background and Aims}

Vedolizumab is a humanized immunoglobulin G1 monoclonal antibody to a $4 \beta 7$ integrin that modulates intestinal, but not brain lymphocyte trafficking. The drug has been found to be more effective than placebo in the induction and maintenance of remission in patients with ulcerative colitis (UC) and Crohn's disease (CD) [1,2]. Unfortunately, some patients only partially respond to therapy and others do not respond at all.

Previous experience with anti-tumor necrosis factor (TNF) drugs has shown that nonresponse or loss of response to therapy is associated with low drug levels and/or the presence of anti-drug antibodies [3, 4]. There have been mixed findings on the association between vedolizumab levels and outcomes, and further prospective studies using objective outcomes and standardized endoscopic scores are needed [5-7]. The aim of this study was to assess the association between serum vedolizumab concentrations (SVC) at several time points through treatment and the presence of antibodies to vedolizumab (ATV) with endoscopic disease activity at week 52 in patients with inflammatory bowel diseases (IBD). We also sought to assess the correlation between SVC through the first 30 weeks of therapy and several variables previously associated with the pharmacokinetics of biologics such as albumin, body mass, inflammatory markers in serum and stool, and the concomitant use of immunomodulators.

\section{Methods}

\section{Subjects and Setting}

We designed a prospective cohort study performed in patients with $\mathrm{CD}$ or UC with active endoscopic disease initiating vedolizumab at the Medical College of Wisconsin/ Froedtert Hospital (Milwaukee, Wisconsin). The study was approved by the local Institutional Review Board, and every patient signed an informed consent. Patients were included if they had active endoscopic disease and were starting therapy with vedolizumab. Active endoscopic disease was defined as a simple endoscopic score for Crohn's disease (SES-CD) > 2 (in CD patients) or an endoscopic Mayo score (EMS) > 1 (in UC patients) [8, 9]. All patients received induction with vedolizumab, $300 \mathrm{mg}$ at weeks 0,2 , and 6 followed by $300 \mathrm{mg}$ every 8 weeks.

We excluded patients with ostomies, history of a colectomy or those with previous exposure to vedolizumab, and patients with no evidence of baseline active endoscopic disease. We also excluded those patients that were lost to follow-up or discontinued vedolizumab for reasons other than lack of efficacy (such as intolerance or lack of insurance coverage). 


\section{Evaluations and Input Variables}

Independent variables considered were demographics, IBD phenotype, smoking status, body mass, previous exposure to biologics, and concomitant medications used for the treatment of IBD. Assessment of endoscopic disease activity was considered at baseline (before starting vedolizumab) and at the time of primary outcome (week 52 [ \pm 8 weeks]). The endoscopist performing the colonoscopies and clinicians caring for each patient were blinded to the serum drug concentrations and antibodies for each patient.

Clinical disease activity was also recorded at baseline, and at the time each serum sample was drawn (just prior to each infusion). The Harvey Bradshaw Index (HBI) was used to assess clinical disease activity in patients with CD [10]. A score $<5$ was considered clinical remission. On those patients with UC, the partial Mayo score (pMS) was used to assess disease [11]. A score $<2$ was considered clinical remission.

Serum samples were drawn just before the vedolizumab infusion at weeks 2, 6, 14, 22, and 30 for measurement of SVC and ATV (unless the drug was discontinued or the patient lost follow-up). C-reactive protein (CRP) and albumin levels were also measured at weeks 0, 14, 30, and 52. Fecal calprotectin (FC) levels were recorded at baseline. Normal serum CRP and albumin levels were defined as per the used assay were $\leq 0.5 \mathrm{mg} / \mathrm{dl}$ and $\geq 3.5 \mathrm{mg} / \mathrm{dl}$, respectively.

The use of IBD medications was documented at every visit. 5-Aminosalicylates were considered if prescribed for 30 or more consecutive days prior to the initiation of vedolizumab. Corticosteroid (CS), including budesonide, was recorded at the time vedolizumab was started and at each follow-up. Rectally administered topical steroids (e.g., enemas or suppositories) were not considered in the analysis. Immunomodulators (azathioprine, mercaptopurine, and methotrexate) were also considered if started concomitantly with vedolizumab or if the patient had been on it before beginning treatment. Previous exposure and response to other biologics were logged at baseline for every patient. Changes in medications (including corticosteroids) were assessed at each study visit.

We also acknowledged vedolizumab dose escalation, which was done as standard of care by the treating provider. Vedolizumab dose escalation was defined as decreasing the interval of drug administration to every 4 weeks. Clinicians managing each patient were blinded to the drug levels, and dose escalation or drug discontinuation was not influenced by pharmacokinetic data.

\section{Measurement of Vedolizumab Levels and Anti-vedolizumab Antibodies}

SVC and ATV were measured using a validated drug-tolerant assay specific for vedolizumab that utilized the previously described homogeneous mobility shift assay (HMSA) methodology [12]. Briefly, vedolizumab levels were measured by calculating the shift in antigen bound vedolizumab complex on a size exclusion column by HPLC. ATV levels were quantified using a size exclusion chromatography-based mobility shift assay, run on a HPLC system with fluorescent detection. The lower limits of quantitation for vedolizumab and ATV were $1.6 \mu \mathrm{g} / \mathrm{ml}$ and $1.6 \mathrm{U} / \mathrm{ml}$, respectively. The upper limits of quantitation for vedolizumab and ATV were $40 \mu \mathrm{g} / \mathrm{ml}$ and $75 \mathrm{U} / \mathrm{ml}$, respectively. The investigators 
conducting the measurements were blinded to the demographic, clinical, and endoscopic data of the patients.

\section{Outcomes}

The primary outcome was steroid-free endoscopic remission at week 52 while on a standard dose of vedolizumab ( \pm 8 weeks). Endoscopic remission was defined as an SES-CD $\leq 2$ in patients with CD and an EMS $\leq 2$ in UC patients while off corticosteroids. Steroid-free was considered as 14 weeks off any oral or IV corticosteroid (including budesonide). Patients that discontinued the drug due to lack of efficacy and/or required dose escalation were considered to have failed the primary outcome.

Secondary outcomes were steroid-free clinical remission at weeks $14,22,30$, and 52, drug discontinuation (stratified as early [within 6 months of drug initiation] and late [after 6 months of drug initiation]) and need for drug dose escalation. Clinical remission was defined as an HBI $<5$ in CD and a pMS $<2$ in UC. Loss of response was defined as having achieved clinical remission at week 22 and then experiencing disease recurrence at any time point through week 52 (HBI $\geq 5 / \mathrm{pMS} \geq 2$, need for new course of steroids due to disease exacerbation and/or discontinuation of the drug due to inefficacy).

\section{Statistical Analysis}

Descriptive statistics were used to examine the baseline characteristics of the study population. Continuous variables were compared using Student's $t$ test or the MannWhitney $U$ test (for nonparametric variables). The Chi-squared test was used to evaluate distributions of categorical variables. Correlation between serum VDZ concentrations and other continuous variables was performed using Spearman's rank correlation test. Interquartile analysis was performed to assess thresholds associated with remission. Receiver operating characteristic (ROC) curves were generated in order to look for association of outcomes and VDZ levels. A $p$ value $<0.05$ was considered statistically significant.

\section{Results}

\section{Patient Characteristics and Outcomes}

Fifty-five patients met inclusion criteria and were followed up for a median of 44 weeks (range of 8-54 weeks). A total of 244 samples were analyzed for those 55 patients (average of 4.4 samples per patient). The baseline characteristics of the study group at the time of vedolizumab initiation are shown in Table 1. Twenty-six patients (47\%) remained on VDZ at week 52, and they had all achieved the primary outcome of steroid-free endoscopic remission at week 52 of therapy (56\% of those with CD and $40 \%$ of those with UC). Thirty (54.6\%), 32 (58.2\%), and 27 (49\%) of patients were in clinical remission at week 14, 22, and 30, respectively. Eight (35\%) of those patients that discontinued vedolizumab did so before week 30 of therapy. Ten patients (18\%) were dose-escalated to $300 \mathrm{mg}$ of vedolizumab every 4 weeks at some point through the follow-up (between weeks 14 and 52). All dose escalations were done due to primary or secondary non-response to the standard dosing. 


\section{Vedolizumab Levels, Anti-drug Antibodies, and Outcomes}

Median SVC were $23.1 \mathrm{mcg} / \mathrm{ml}$ at week 2 (IQR 19.4-25.8), $19.8 \mathrm{mcg} / \mathrm{ml}$ at week 6 (IQR 12.4-26.4), $9.2 \mathrm{mcg} / \mathrm{ml}$ at week 14 (IQR 6-16), $6.9 \mathrm{mcg} / \mathrm{ml}$ at week 22 (IQR 3.6-12), and $5.8 \mathrm{mcg} / \mathrm{ml}$ at week 30 (IQR 4.1-11). Patients that achieved steroid-free endoscopic remission by week 52 had higher SVC at weeks 2, 6, 14, 22, and 30, but only achieved statistical significance at weeks 2 and 6 (Fig. 1 and Table 2). Patients in clinical remission at week 52 had significantly higher median SVC at week 2 when compared to those that were not (23.8 [IQR 19-32] vs. 19.8 [IQR $15-23] \mathrm{mcg} / \mathrm{ml}, p<0.01$ ). Those who achieved clinical remission at week 30 demonstrated higher SVC at weeks 2,6 , and 14, with only weeks 2 and 6 reaching statistical significance ( $p=0.01$ and $p=0.02$, respectively). There were no significant differences in SVC throughout induction between those patients that were and were not escalated during maintenance. When comparing SVC between patients with UC and $\mathrm{CD}$, there was an overall trend toward higher SVC on those patients with $\mathrm{CD}$ versus those with UC even though the difference only reached statistical significance at week 2 (24.2 [IQR 21.7-29.3] for CD and 22.5 [IQR 17.6-25.3] for UC, $p=0.04-$ Supplemental Table 1).

When measuring area under the curve, we found good correlation between SVC at weeks 2 and 6, and endoscopic remission at weeks 52 (ROC: 0.72 [ $p=0.02]$ and ROC: 0.69 [ $p=$ 0.023], respectively). We also found a good correlation between SVC at week 6 and clinical remission at week 14 (ROC: $0.7[p=0.02]$ ). Furthermore, there was a significant association between SVC at week 2 and clinical remission at weeks 30 and week 52 (ROC: 0.73 [ $p=$ $0.015]$ and ROC: $0.72[p=0.014])$.

Only 3 of the 55 study subjects (5.5\%) had detectable ATV at some point through the follow-up. One had detectable antibodies at week 2 that became undetectable at week 6 with no recurrence at week 14 (vedolizumab was discontinued due to non-response at week 20). The two other patients had detectable antibodies at week 2 and became undetectable at week 6. Neither of these patients was in remission at week 52.

\section{Vedolizumab Pharmacokinetics in Patients That Were Dose-Escalated}

We also explored the pharmacokinetics of vedolizumab in those patients that were doseescalated. Ten patients were dose-escalated from $300 \mathrm{mg}$ every 8 weeks to $300 \mathrm{mg}$ every 4 weeks. Of those, 6 had trough levels measured before and after dose escalation (all of them had UC). The median SVC before and after dose escalation were $6.9 \mathrm{mcg} / \mathrm{ml}$ (IQR 3.8-20.2) and $17.2 \mathrm{mcg} / \mathrm{ml}$ (IQR 12.2-34.6), respectively. The median increase in SVC was 9.1 $\mathrm{mcg} / \mathrm{ml}$ (IQR 7-15.7). Two of those patients (33\%) did achieve endoscopic remission by week 52 .

\section{Vedolizumab Drug Concentrations and Loss of Response}

Five patients (9\%) lost clinical response throughout follow-up. Only one of those patients was in endoscopic remission despite the presence of symptoms. Those patients that had achieved clinical remission at week 22 but lost clinical response by week 52 have significantly lower median SVC at week 14 when compared to those that did not lose response (6 [IQR 3-8.8] and 11.2 [IQR 5.7-16.7], respectively, $p=0.02$ ). The difference in 
SVC at other time points was not statistically significant. However, those patients that lost response had numerically lower concentration levels throughout the maintenance phase of therapy (Supplemental Table 2).

\section{Biomarkers, Body Mass, and Vedolizumab Pharmacokinetics}

Overall, there was a negative correlation between baseline (week 0) CRP and SVC throughout the study period, even though only the levels at week 14 achieved statistical significance (Table 3). There was a positive correlation between baseline serum albumin and SVC at every time point except for week 2 (Table 3). There was also an inverse correlation between baseline fecal calprotectin levels and SVC, even though it only reached statistical significance at week 2 (rho: $-0.43, p=0.002$ ) and week 6 (rho: $-0.31, p=0.03$ ).

We did not find a correlation between body mass and SVC [weeks 2 (rho: $-0.34, p=0.01$ ), week 6 (rho: $-0.24, p=0.08$ ), week 14 (rho: $-0.12, p=0.39$ ), week 22 (rho: $-0.16, p=$ 0.31 ) and week 30 (rho: $-0.25, p=0.21$ )]. Moreover, we did not find a correlation between age and SVC at any time point $(p>0.05$ for all).

\section{Cross-Sectional Correlation Between Vedolizumab Levels, Biomarkers, and Clinical Outcomes at Different Time Points of Maintenance Therapy}

There was no significant difference in median SVC between patients in clinical remission and active disease at weeks 14 (11.2 [IQR 5.8-16.8] vs. 8.5 [IQR $6.2-12.1] \mathrm{mcg} / \mathrm{ml}, p=$ 0.32 ), week 22 (8.8 [IQR 3.9-11.7] vs. 5.3 [IQR 3.5-12] mcg/ml, $p=0.7)$ and week 30 (8.4 [IQR 5.1-10.5] vs. 5.2 [IQR 3.3-15.3] $\mathrm{mcg} / \mathrm{ml}, p=0.8$ ). We found a statistically significant negative correlation between SVC and CRP at week 14 (rho: $-0.34, p=0.039$ ) and week 22 (rho: $-0.37, p=0.03$ ) but not at week 30 (rho: $-0.3, p=0.21$ ). We also found a relationship between SVC and serum albumin at week 14 (rho: $0.55, p<0.001$ ) but not at week 22 (rho: $0.23, p=0.25$ ) or week 30 (rho: $0.5, p=0.09$ ).

Given that we found a link between disease remission and SVC at weeks 2 and 6, we sought to find threshold levels that best predicted disease remission at those time points. Interquartile values for serum VDZ concentrations at week 2 were 19.4, 23.1 and 25.8 $\mathrm{mcg} / \mathrm{ml}$ (Fig. 2). SVC $\geq 23.2 \mathrm{mcg} / \mathrm{ml}$ at week 2 (the interquartile value between quartiles 2 and 3) were associated with endoscopic remission at week 52 (OR 8.8 [95\% CI 2.6-29.7], $p$ $<0.001$-Fig. 2) and clinical remission at week 14 (OR 3.1 [95\% CI 1.01-9.3], $p=0.044$ ), week 22 (OR 6.3 [95\% CI 1.9-20.7], $p=0.002$ ) and week 30 (OR 5.0 [95\% CI 1.6-15.8], $p$ $<0.01$ ). A multivariate analysis adjusting for potential confounder factors such as type of disease (UC versus CD), baseline albumin level, body mass, combination therapy with an immunomodulator, smoking status, and baseline CRP and FC levels was performed. We found that an SVC $\geq 23.2 \mathrm{mcg} / \mathrm{ml}$ at week 2 remained significantly associated with remission at week 52 (OR 9.1 [95\% CI 1.3-4.1], $p<0.01$ ). The only other variable that resulted significant in the multivariate analysis was baseline hypoalbuminemia, defined as a level $<3.8 \mathrm{~g} / \mathrm{dl}$ per local laboratory (OR 14 [95\% CI 1.7-11.9], $p=0.015$ ).

The interquartile SVC level values at week 6 were 12.5, 19.8, and $26.4 \mathrm{mcg} / \mathrm{ml}$ (Fig. 3). We found that a serum VDZ concentration $\geq 19.8 \mathrm{mcg} / \mathrm{ml}$ at week 6 (the interquartile value between quartiles 2 and 3) was also associated with endoscopic remission at week 52 (OR 
3.1 [95\% CI 1.02-9.3], $p=0.04$-Fig. 3). A SVC $\geq 19.8 \mathrm{mcg} / \mathrm{ml}$ at week 6 was also associated with clinical remission at week 14 (OR 6.0 [95\% CI 1.9-19.4], $p=0.002$ ), week 22 (OR 4.4 [95\% CI 1.4-13.8], $p=0.01$ ), and week 30 (OR 7.1 [95\% CI 2.2-23.5], $p<$ 0.001 ). As with threshold levels at week 2 , we performed multivariate analysis adjusting for the confounding factors listed above. In this adjusted analysis, a serum VDZ concentration $\geq$ $19.8 \mathrm{mcg} / \mathrm{ml}$ at week 6 was also associated with remission at week 52 (OR 6.8 [95\% CI 1.24], $p=0.033)$. As with the analysis at week 2 , hypoalbuminemia was also independently associated with remission at week 52 (OR 13 [95\% CI 1.9-11.9], $p=0.015$ ).

\section{Combination Therapy, Thiopurine Metabolites, and Vedolizumab Levels}

Twenty patients were on combination therapy ( 3 on methotrexate and 17 on a thiopurine). Patients on combination therapy with an immunomodulator did not have notable differences in SVC at weeks 2, 6, 14, 22, or 30 (Fig. 4). Even though the analysis was limited due to the low number of patients on azathioprine/mercaptopurine, there was no significant correlation between thiopurine drug dose and SVC at week 2 (rho: $-0.05[p=0.843]$ ), week 6 (rho: 0.024 [ $p=0.92]$ ), week 14 (rho: $0.32[p=0.19]$ ), week 22 (rho: $0.5[p=0.14]$ ) or week 30 (rho: $0.37[p=0.3]$ ). Furthermore, there was no correlation between 6-thioguanine levels measured between weeks 14 and 30 and SVC at any time point ( $p>0.05$ for all).

\section{Discussion}

Therapeutic drug monitoring (TDM) has emerged as a tool to manage patients with IBD receiving anti-TNF drugs. The role of TDM in the setting of biologic IBD therapies with other mechanisms of actions has yet to be defined. In this study, we found a correlation between SVC during induction therapy and endoscopic remission at week 52 of treatment, even though there was no significant correlation between those SVC at weeks 14 and 30 and outcomes at week 52 .

These results may help to identify early in the course of treatment (during induction), those patients who are more likely to have a good long-term response to vedolizumab. Our findings are important as they may imply that optimizing vedolizumab levels during early in the course of therapy may positively impact disease remission after 1 year. Although there was a trend toward higher trough SVC during maintenance therapy (week 14 and 30) on those patients that achieved endoscopic remission at week 52, it did not reach statistical significance. One potential explanation for this is the lack of statistical power to assess difference during maintenance.

Other studies looking into clinical and endoscopic outcomes have had similar (but not exactly the same) results. Our study has the strength of having prospectively collected baseline and follow-up endoscopic disease activity on all patients using standardized and validated scores (SES-CD and EMS). Moreover, we used a drug-tolerant HMSA to measure drug levels and ATV. Yacoub et al. found that vedolizumab levels at week 6 were significantly higher on those patients that responded to therapy after a year [7]. Ungar et al found an association between serum VDZ concentrations and clinical disease activity at week 6 [5]. Analysis from the GEMINI I and II pivotal studies also showed that response and remission to therapy were achieved at a higher rate on those patients with higher SVC 
$[1,2]$. Dreesen et al. found that those patients who achieved endoscopic remission at week 14 in UC and week 22 in CD had higher SVC at week 6, but not at week 2 [6]. Disparities between studies may be explained by differences in timing of endoscopy and the definition of remission used. Other studies used "lack of ulcerations" as remission in $C D$, while we used standardized endoscopic scores to assess mucosal healing (SES-CD). Another relevant difference among studies is the use of different assays. However, even when considering these disparities, the overall trend is to find more significant differences in SVC between responders and non-responders early in the course of therapy (induction).

An important question with studies looking into the correlation between vedolizumab drug exposure and outcomes is why an association between drug exposure and efficacy has been seen even though some studies have shown that only low doses of vedolizumab are needed to completely saturate the integrin receptor of $\mathrm{T}$ cells $[5,13]$. The exact explanation for these findings is not clear. However, we do know that the rate of integrin saturation is not correlated with disease response and higher doses of vedolizumab correlated with better clinical outcomes [14]. A potential explanation is an alternative or additional mechanism of action for vedolizumab, which do require higher doses.

As in other studies, we found a very low rate of immunogenicity. In our cohort, only three patients developed detectable ATV and they were all transient. Immunogenicity has been an important factor explaining non-response in patients receiving anti-TNF [15]. With anti-TNF drugs the rate of immunogenicity increases with time exposed to drug and with interruptions of therapy. The low incidence found in this study may be due to the relatively shorter followup after drug initiation. The HMSA used in this study is drug tolerant and can detect ATV in the presence of the drug; therefore, this variable would not explain the low rate of immunogenicity. Studies looking into the presence of ATV in patients with long-term exposure to vedolizumab, especially those that have had interruptions in treatment, are needed.

We also found that baseline albumin levels had a significant correlation with drug levels throughout the follow-up period. Mechanistically this may be because serum albumin and monoclonal antibodies share a similar metabolism pathway. [16]. Albumin has also been found to be correlated with SVC in previous studies looking into the pharmacokinetics of vedolizumab $[5,7,17]$.

While it was only an exploratory outcome and the analysis was limited by the low number of patients, it was remarkable to find that those patients that achieve clinical remission by week 22 and then lost response between weeks 22 and 52 had lower SVC during maintenance (but not during induction). This may support the use of reactive TDM and potentially dose escalation to recapture response when patients develop secondary non-response. In the GEMINI trials, more than 50\% of patients who lost response to vedolizumab on a every 8week dosing schedule achieved clinical response after an increase in dose to every 4 weeks. Randomized trials looking into dose escalation after secondary non-response are warranted.

Related to this last point, we sub-analyzed levels before and after vedolizumab dose optimization. We found that SVC did significantly increase when compared before and after 
dose optimization. In a subgroup analysis, Williet et al. did not find a difference [18]. The pharmacokinetic profile of those patients that undergo vedolizumab dose optimization is warranted.

Limitations of our study include a low power to detect smaller differences in levels during the maintenance period. While the SVC were numerically higher, they did not achieve statistical significance. However, at a larger scale, a difference could have been discernible. As with other studies looking into biologic drug levels and disease activity, we need to take into account that this is an observational analysis and other factors can explain these findings. For example, those patients with a lower rate of response may have higher drug clearance due to a higher burden of systemic inflammation and/or a higher drug loss through the gastrointestinal tract as is seen with other biologics [19].

In conclusion, we found an association between SVC at induction and endoscopic remission at week 52. This correlation was stronger at week 2 . We also found a very low incidence of immunogenicity against the drug with only 3 patients developing detectable ATV (all of which became undetectable by week 14). Interventional studies looking into higher drug doses during induction may improve long-term outcomes are needed.

\section{Supplementary Material}

Refer to Web version on PubMed Central for supplementary material.

\section{Acknowledgment}

This project was supported by the National Center for Advancing Translational Sciences, National Institutes of Health, Award Number UL1TR001436, and funded by Prometheus Laboratories.

Funding This study was funded by Prometheus Laboratories Inc.

Compliance with ethical standards

Conflict of interest Andres J. Yarur, MD is a consultant, has received research support and is part of the speaker bureau for Takeda pharmaceuticals, Abbvie and Prometheus Laboratories. Alexandra Bruss, CRC, Caroline Fox, PAC, Amir Patel MD and Brandon Berens CRC have no conflict of interests to report. Poonam Beniwal-Patel, MD is part of the speaker bureau of Takeda pharmaceuticals. Snehal Naik, $\mathrm{PhD}$, Anjali Jain $\mathrm{PhD}$ and Bayda Bahur are employees of Prometheus Laboratories. Ryan Ungaro, MD has been part of consulting/advisory board for Takeda, Janssen and Pfizer. He has also received research grant support from Abbvie. Marla Dubinsky, MD is a consultant for Takeda pharmaceuticals and Prometheus Laboratories. Daniel J. Stein, MD is part of the speaker bureau for Takeda pharmaceuticals, Janssen and Abbvie.

\section{References}

1. Feagan BG, Rutgeerts P, Sands BE, et al. Vedolizumab as induction and maintenance therapy for ulcerative colitis. N Engl J Med. 2013;369:699-710. [PubMed: 23964932]

2. Sandborn WJ, Feagan BG, Rutgeerts P, et al. Vedolizumab as induction and maintenance therapy for Crohn's disease. N Engl J Med. 2013;369:711-721. [PubMed: 23964933]

3. Maser EA, Villela R, Silverberg MS, Greenberg GR. Association of trough serum infliximab to clinical outcome after scheduled maintenance treatment for Crohn's disease. Clinical Gastroenterology and Hepatology. 2006;4:1248-1254. [PubMed: 16931170]

4. Yarur AJ, Jain A, Hauenstein SI, et al. Higher adalimumab levels are associated with histologic and endoscopic remission in patients with Crohn's disease and ulcerative colitis. Inflamm Bowel Dis. 2016;22:409-415. [PubMed: 26752470] 
5. Ungar B, Kopylov U, Yavzori M, et al. Association of vedolizumab level, anti-drug antibodies, and a $4 \beta 7$ occupancy with response in patients with inflammatory bowel diseases. Clin Gastroenterol Hepatol. 2018;16:697-705e7. [PubMed: 29223444]

6. Dreesen E, Verstockt B, Bian S, et al. Evidence to support monitoring of vedolizumab trough concentrations in patients with inflammatory bowel diseases. Clin Gastroenterol Hepatol. 2018;16:1937-1946e8. [PubMed: 29704680]

7. Yacoub W, Williet N, Pouillon L, et al. Early vedolizumab trough levels predict mucosal healing in inflammatory bowel disease: a multicentre prospective observational study. Aliment Pharmacol Ther. 2018;47:906-912. [PubMed: 29384209]

8. Daperno M, D’Haens G, Van Assche G, et al. Development and validation of a new, simplified endoscopic activity score for Crohn's disease: the SES-CD. Gastrointest Endosc. 2004;60:505-512. [PubMed: 15472670]

9. Schroeder KW, Tremaine WJ, Ilstrup DM. Coated oral 5-aminosalicylic acid therapy for mildly to moderately active ulcerative colitis. A randomized study. N Engl J Med. 1987;317:1625-1629. [PubMed: 3317057]

10. Harvey RF, Bradshaw JM. A simple index of Crohn's-disease activity. Lancet. 1980;1:514. [PubMed: 6102236]

11. Lewis JD, Chuai S, Nessel L, Lichtenstein GR, Aberra FN, Ellenberg JH. Use of the noninvasive components of the mayo score to assess clinical response in Ulcerative Colitis. Inflamm Bowel Dis. 2008;14:1660-1666. [PubMed: 18623174]

12. Wang S-L, Ohrmund L, Hauenstein S, et al. Development and validation of a homogeneous mobility shift assay for the measurement of infliximab and antibodies-to-infliximab levels in patient serum. J Immunol Methods. 2012;382:177-188. [PubMed: 22691619]

13. Wyant T, Estevam J, Yang L, Rosario M. Development and validation of receptor occupancy pharmacodynamic assays used in the clinical development of the monoclonal antibody vedolizumab. Cytometry. 2015;90:168-176. [PubMed: 25908521]

14. Yarur AJ, Deshpande AR, Pechman DM, Tamariz L, Abreu MT, Sussman DA. Inflammatory bowel disease is associated with an increased incidence of cardiovascular events. Am J Gastroenterol. 2011;106:741-747. [PubMed: 21386828]

15. Afif W, Loftus EV, Faubion WA, et al. Clinical utility of measuring infliximab and human antichimeric antibody concentrations in patients with inflammatory bowel disease. Am J Gastroenterol. 2010;105:1133-1139. [PubMed: 20145610]

16. Yarur AJ, Rubin DT. Therapeutic drug monitoring of anti-tumor necrosis factor agents in patients with inflammatory bowel diseases. Inflamm Bowel Dis. 2015;21:1709-1718. [PubMed: 25901974]

17. Rosario M, Dirks NL, Gastonguay MR, et al. Population pharmacokinetics-pharmacodynamics of vedolizumab in patients with ulcerative colitis and Crohn's disease. Aliment Pharmacol Ther. 2015;42:188-202. [PubMed: 25996351]

18. Williet N, Boschetti G, Fovet M, et al. Association between low trough levels of vedolizumab during induction therapy for inflammatory bowel diseases and need for additional doses within 6 months. Clin Gastroenterol Hepatol. 2017;15:1750-1757e3. [PubMed: 27890854]

19. Brandse JF, van den Brink GR, Wildenberg ME, et al. Loss of infliximab into feces is associated with lack of response to therapy in patients with severe ulcerative colitis. Gastroenterology. 2015;149:350-355e2. [PubMed: 25917786] 


\section{Association Between Vedolizumab Levels and Remission at Week 52}

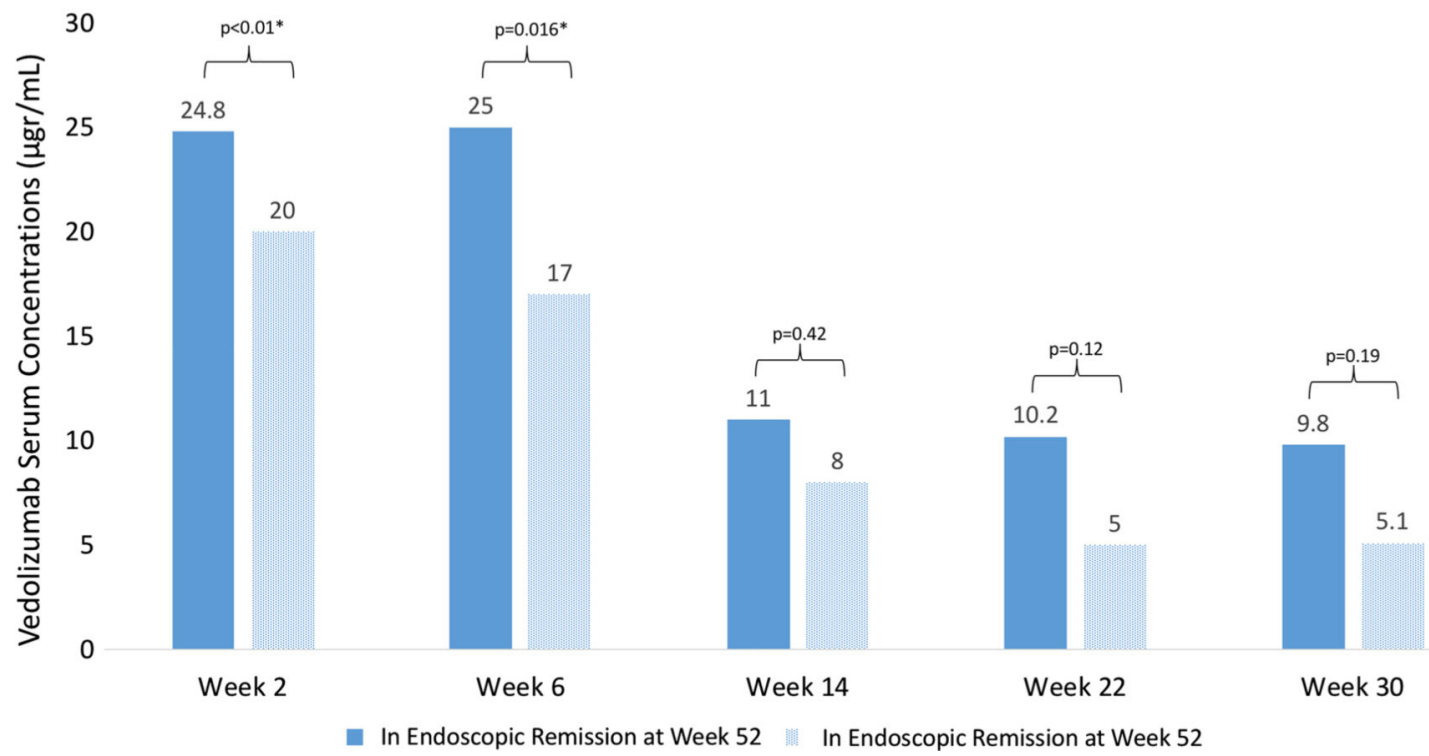

Fig. 1.

Differences between vedolizumab levels at several time points among patients that were and were not in remission at week 52 of therapy. Endoscopic remission was defined as mucosal healing, clinical remission, and normal C-reactive protein 


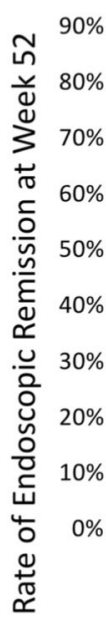

\%

$80 \%$

$70 \%$

$60 \%$

$50 \%$

$40 \%$

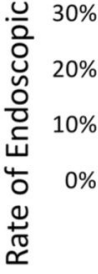

$31 \%$

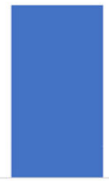

Quartile I

$0-19.4 \mathrm{mcg} / \mathrm{ml}$

$79 \%$

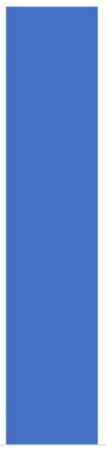

Quartile III

$23.2-25.8 \mathrm{mcg} / \mathrm{ml}$

$25.9-34.4 \mathrm{mcg} / \mathrm{m}$

Serum Vedolizumab Level at Week 2 of Therapy by Quartile

Fig. 2.

Rates of remission at week 52 by serum vedolizumab quartile level at week 2 
กิ $80 \%$

这 $70 \%$

3

$\sum_{\pi}^{+} 60 \%$

으 $50 \%$

岕 $40 \%$

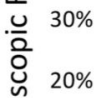

응 $20 \%$

ए $10 \%$

잉

$\stackrel{乛}{\check{x}}$

\section{Quartile I}

$0-12.4 \mathrm{mcg} / \mathrm{ml}$
$23 \%$

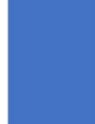

Serum Vedolizumab Level at Week 6 of Therapy by Quartile

$p$ value for trend $=0.09$

Fig. 3.

Rates of remission at week 52 by serum vedolizumab quartile level at week 6 
Differences Between Patient On Combination Therapy and Monotherapy

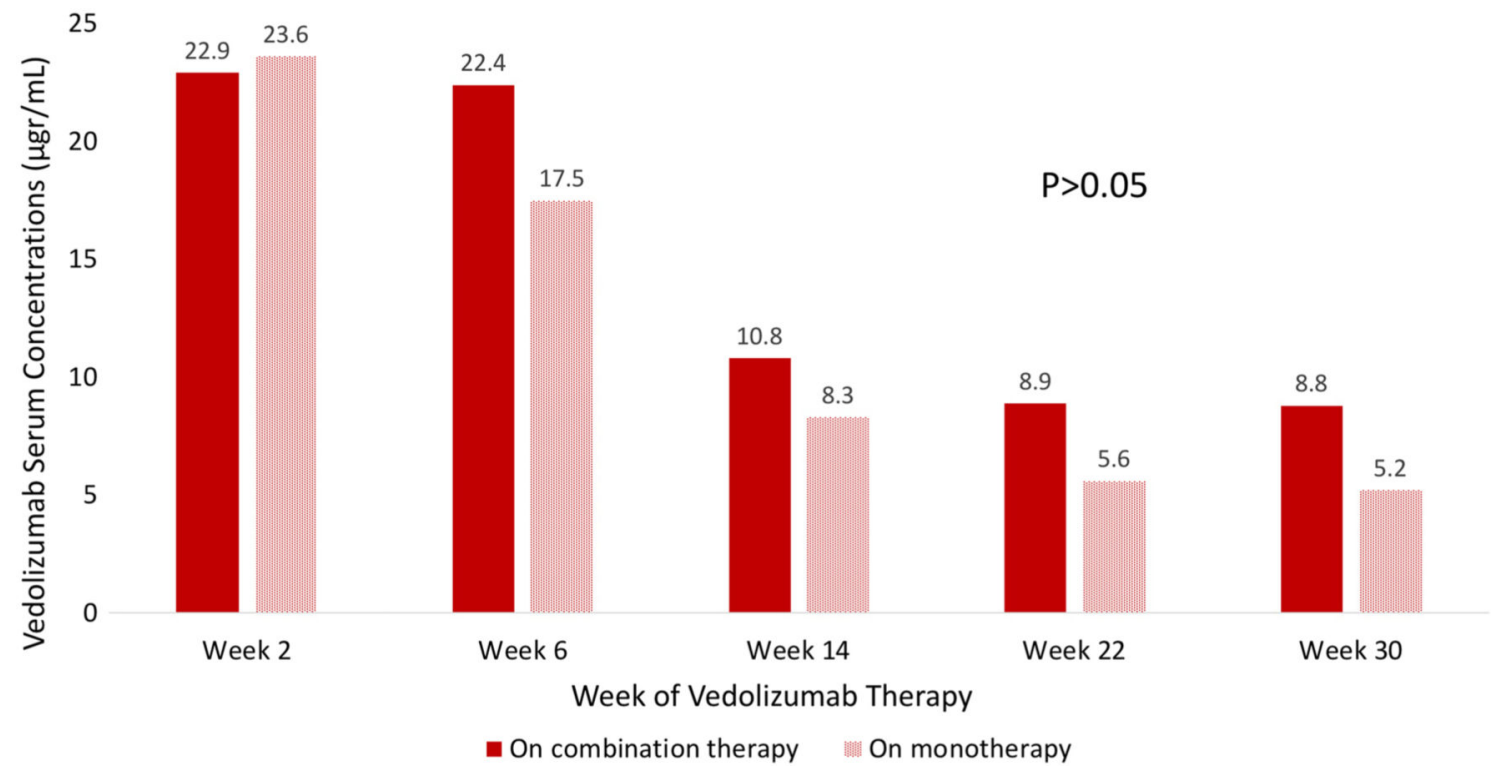

Fig. 4.

Differences in vedolizumab concentrations through week 30 of treatment between those patients that were on monotherapy with vedolizumab versus those that received combination therapy with vedolizumab and a immunomodulator 


\section{롤

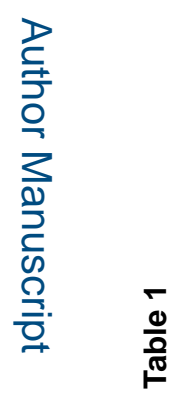

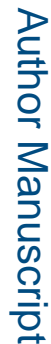

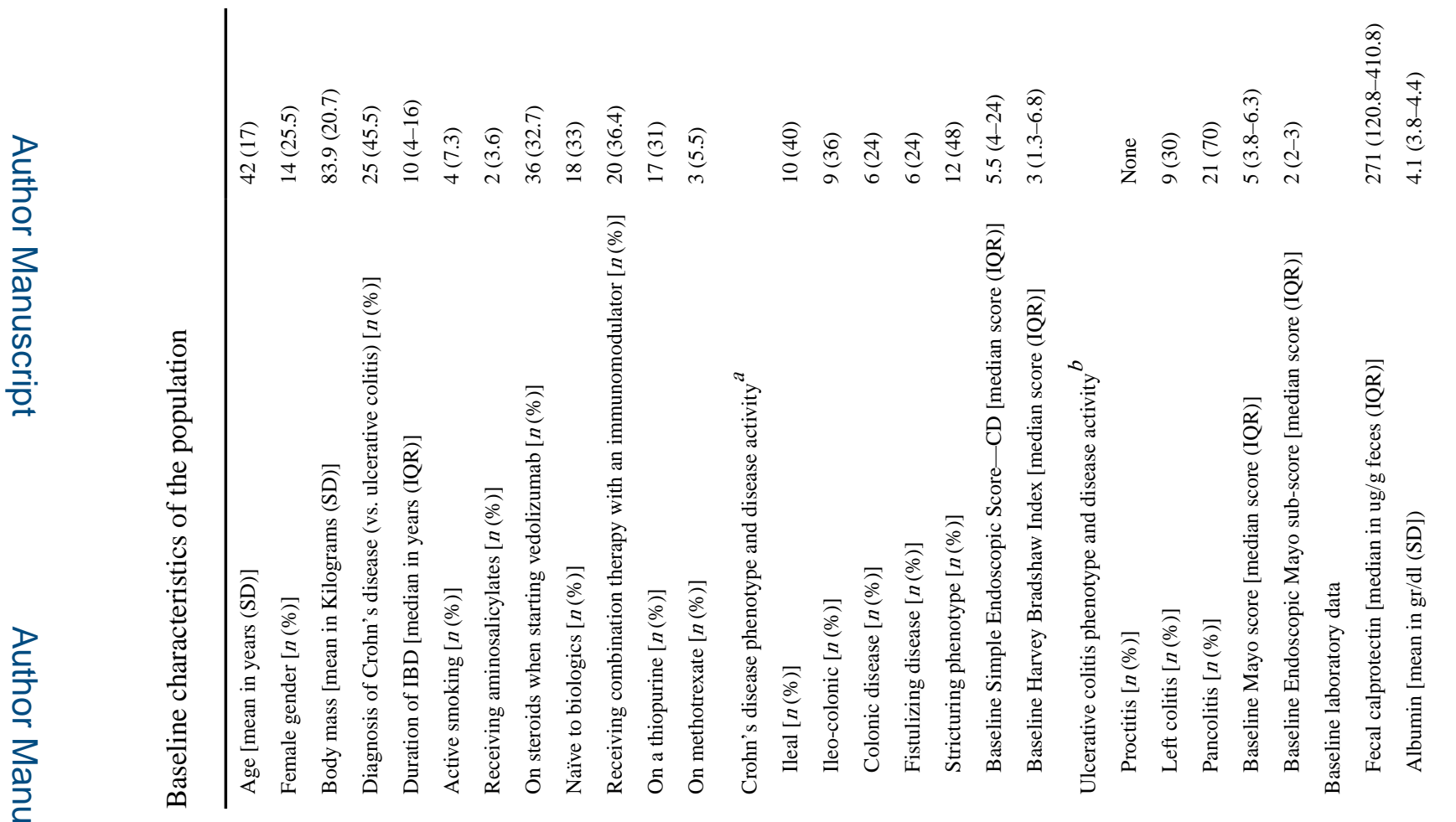

Dig Dis Sci. Author manuscript; available in PMC 2020 March 05. 


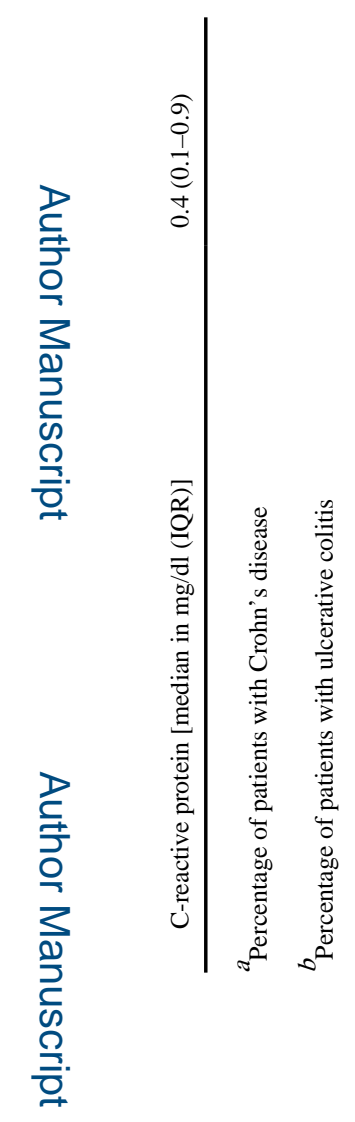

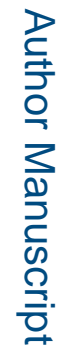

로을

Dig Dis Sci. Author manuscript; available in PMC 2020 March 05. 


\section{롤 \\ }

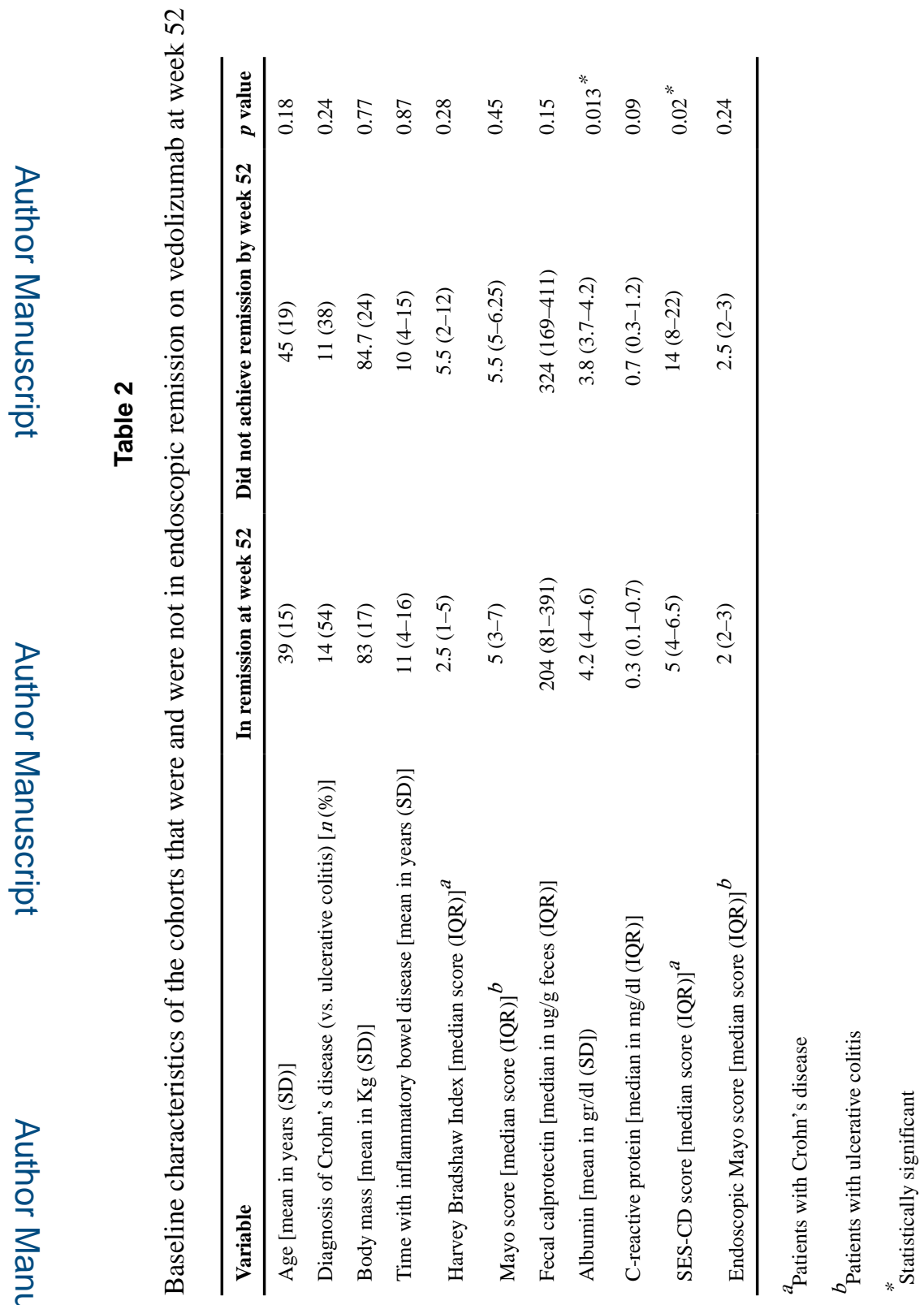

Dig Dis Sci. Author manuscript; available in PMC 2020 March 05. 


\section{롤 \\ 일}

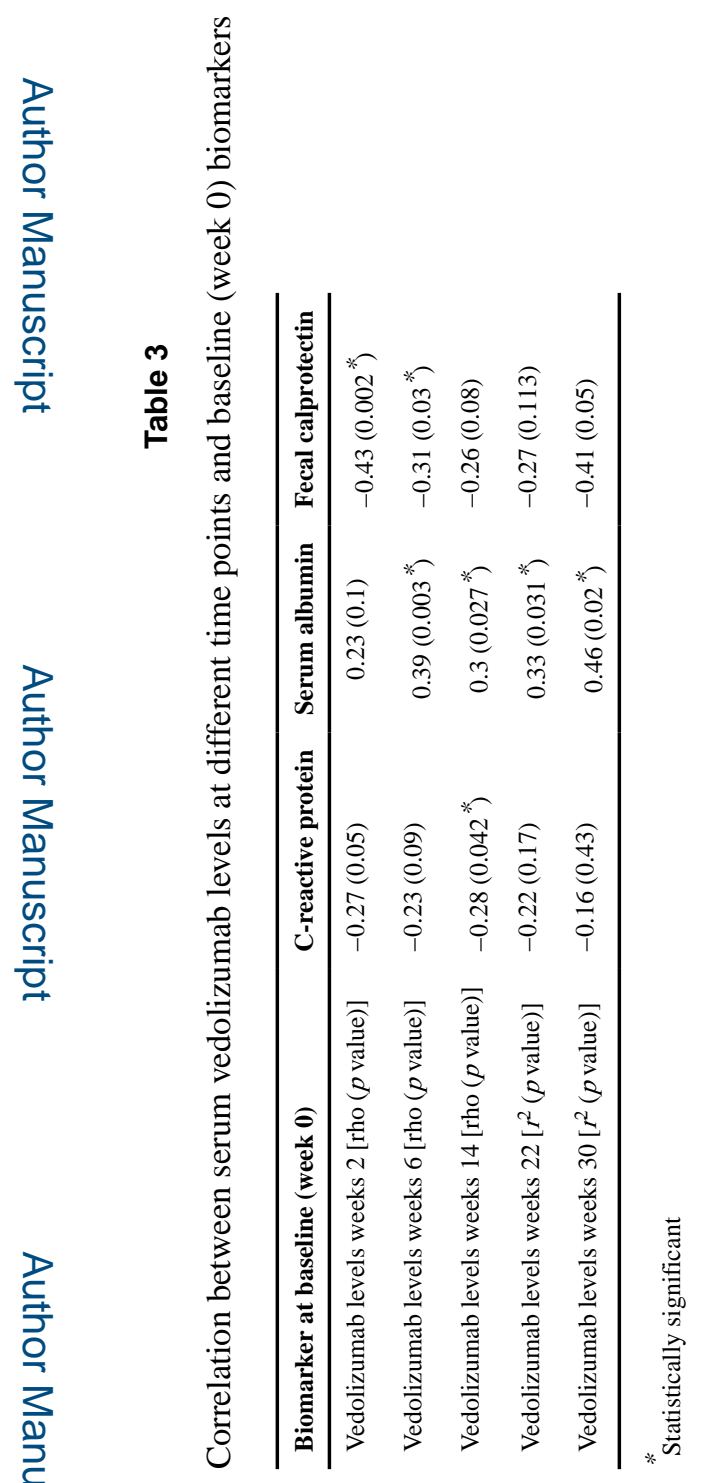

Dig Dis Sci. Author manuscript; available in PMC 2020 March 05. 